\title{
Doença Arterial Coronariana em Pacientes com Valvopatia Reumática e Não-Reumática Acompanhados em Hospital Público do Rio de Janeiro
}

\author{
Coronary Artery Disease in Patients with Rheumatic and Non-Rheumatic Valvular Heart Disease Treated at a \\ Public Hospital in Rio de Janeiro
}

Dany David Kruczan, Nelson Albuquerque de Souza e Silva, Basílio de Bragança Pereira, Vítor André Romão, Wilson
Braz Correa Filho, Fidel Ernesto Castro Morales
Instituto Estadual de Cardiologia do Rio de Janeiro Aloysio de Castro/IECAC - Universidade Federal do Rio de Janeiro/UFRJ - Rio de Janeiro, RJ - Brasil

Resumo

Objetivo: Avaliar a prevalência de doença arterial coronariana (DAC) na valvopatia de etiologia reumática e nãoreumática, examinando possíveis fatores preditivos da presença da doença.

Métodos: Estudo transversal, de série de casos obtidos em população pré-definida. Foram avaliados 1.412 pacientes com indicação de cirurgia cardíaca por qualquer etiologia. Destes, foram encontrados e estudados 294 casos com valvopatia primária de etiologias reumática e não-reumática, com idade $\geq 40$ anos, submetidos a coronariografia.

Resultados: Os valvopatas reumáticos apresentaram menor prevalência de DAC (4\%) que os não-reumáticos (33,61\%) ( $p<0,0001$ ). O modelo de regressão logística evidenciou que idade, dor torácica típica, hipertensão arterial sistêmica (HAS), diabete melito e dislipidemia estavam significativamente relacionados à DAC, e que a etiologia reumática não era determinante da doença. Tabagismo e sexo revelaram-se de importância clínica na DAC, embora sem significância estatística. No grupo total, o modelo de análise Log linear demonstrou que, independentemente da etiologia, sexo, idade $\geq 55$ anos, HAS, dor torácica típica, diabete e dislipidemia se relacionavam diretamente com a DAC, sendo as três últimas as variáveis de maior peso para a doença.

Conclusão: A prevalência de DAC é baixa entre valvopatas reumáticos e mais alta entre não-reumáticos; a etiologia reumática não parece exercer efeito protetor sobre a prevalência de DAC; e as variáveis sexo, idade, HAS, dor torácica típica, dislipidemia e diabete melito foram identificadas como fortemente associadas à presença de DAC. É possível definir critérios de indicação de coronariografia pré-operatória nas trocas valvares, podendo-se evitar a indicação rotineira a partir dos 40 anos. (Arq Bras Cardiol 2008;90(3):217-223)

Palavras-chave: Arteriosclerose coronariana/epidemiologia, cardiopatia reumática/diagnóstico, doenças das valvas cardíacas/diagnóstico, angiografia coronariana.

\footnotetext{
Summary

Objective: to estimate the prevalence of coronary artery disease (CAD) in valvular heart disease of rheumatic (RVHD) and non-rheumatic (NVHD) etiology, assessing possible predictive factors for the presence of CAD.
}

Methods: This is a cross-sectional study of a series of cases obtained from a pre-defined population, wherein 1,412 patients referred for heart surgery of any etiology were evaluated. Of these, 294 primary heart disease patients aged $\geq 40$ submitted to cinecoronary arteriography (CA) were identified and studied.

Results: patients with RVHD presented lower prevalence of CAD (4\%) when compared to NVHD (33.61\%), p<0.0001. The logistic regression analysis showed that age, typical angina-like chest pain (TACP), systemic arterial hypertension (SAH), diabetes and dyslipidemia were significantly related to $C A D$, and that the rheumatic etiology was not a disease determinant. Smoking and gender were clinically important in CAD, although not statistically significant. In the whole group, the Log-linear analysis showed that, regardless of the etiology, gender, age $\geq 55$, SAH, TACP, diabetes and dyslipidemia were all related directly to CAD, with the latter three being the most important variables for the disease.

Conclusion: the prevalence of CAD among RVHD patients is low, whereas it is high among NVHD patients; the rheumatic etiology does not seem to have any beneficial effects on the prevalence of CAD; gender, age, SAH, TACP, dyslipidemia and diabetes were identified as being strongly associated with the presence of CAD. It is possible to define the criteria that indicate the need for pre-surgical CA in heart valve replacements, so that the standard indication after the age of 40 years can be avoided. (Arq Bras Cardiol 2008;90(3):197-203)

Key words: Coronary arteriosclerosis/epidemiology; rheumatic heart disease/diagnosis; heart valve diseases/diagnosis; coronary angiography.

Full texts in English - http://www.arquivosonline.com.br

Correspondência: Dany David Kruczan •

Avenida Nossa Senhora de Copacabana, 195/1106 - Copacabana - 22020-002 - Rio de Janeiro, RJ - Brasil

E-mail: dkruczan@cardiol.br, kruczan@centroin.com.br

Artigo recebido em 13/5/07; revisado recebido em 1/8/07; aceito em 8/10/07. 


\section{Introdução}

A coronariografia tem sido indicada como procedimento de rotina na avaliação pré-operatória de pacientes com valvopatias que preenchem os seguintes critérios: homens com idade $\geq 35$ anos, mulheres na pré-menopausa com idade $\geq 35$ anos e com fatores de risco cardiovascular, e mulheres na pós-menopausa ${ }^{1,2}$. A conduta tem como justificativa o diagnóstico de obstruções coronarianas, especialmente as assintomáticas, e a prevalência acentuada de doença arterial coronariana nesses pacientes. O diagnóstico de obstrução coronariana associada indicaria revascularização miocárdica no momento da troca valvar, independentemente da apresentação clínica e da presença ou não de sintomas. Em nosso meio, a coronariografia tem sido indicada quase que obrigatoriamente a partir dos 40 anos. No entanto, na prática clínica observa-se baixa prevalência de doença arterial coronariana entre valvopatas, principalmente os de etiologia reumática. Vale notar que, embora a etiologia nãoreumática das valvopatias seja freqüentemente encontrada em nossa população, a etiologia reumática ainda supera consideravelmente a primeira ${ }^{3,4}$.

Este estudo, desenvolvido em razão dessas observações e com restrições quanto à racionalidade adotada para as indicações, muitas vezes até indiscriminadas, de um método caro, invasivo e não isento de morbidade, teve como objetivos: avaliar a prevalência de doença arterial coronariana em pacientes com lesões orovalvares de etiologia reumática e não-reumática com idade $\geq 40$ anos, encaminhados para avaliação de indicação cirúrgica de troca valvar em hospital público especializado em Cardiologia do Rio de Janeiro; e identificar possíveis fatores preditivos da presença de doença arterial coronariana nesses pacientes, visando examinar a influência da etiologia reumática na doença.

O presente estudo foi realizado com base em observações clínicas nos últimos vinte e cinco anos de atividades em hospital público do Rio de Janeiro. Durante esse período, adquiriu-se vasta experiência no atendimento de pacientes orovalvares, em particular adultos com idade $\geq 40$ anos, de nível socioeconômico baixo, muitos com histórico de surtos agudos de febre reumática, encaminhados para avaliação de indicação cirúrgica de troca valvar, todos com coronariografia.

\section{Métodos}

Em estudo transversal de série de casos realizado em população pré-definida entre 1999 e 2005, foram avaliados 1.412 pacientes encaminhados com indicação de cirurgia cardíaca por qualquer etiologia. Entre esses, foram encontrados e selecionados para a pesquisa 294 pacientes com lesões primárias das valvas cardíacas, sendo 175 de etiologia reumática e 119 de etiologia não-reumática, com idade $\geq 40$ anos, previamente avaliados com coronariografia. Todos os pacientes preenchiam critérios clínicos para os diagnósticos de estenose, insuficiência ou dupla lesão das valvas aórtica e/ou mitral, respaldados em anamnese e em exame clínico ${ }^{5}$. A ecocardiografia foi utilizada para definir os critérios de etiologias reumática e não-reumática ${ }^{6}$.

Para definição da cardiopatia valvar reumática foram considerados os seguintes critérios: 1) na valva mitral, espessamento das bordas livres dos folhetos com ou sem fusão comissural e do aparelho subvalvar com redução da mobilidade e fixação do folheto posterior; e 2) na valva aórtica, espessamento das cúspides, da borda para a base. Para a cardiopatia valvar não-reumática foram considerados os seguintes critérios de doença degenerativa: 1) na valva mitral, espessamento dos folhetos com mobilidade preservada em borda livre; 2) na valva aórtica, degeneração fibrocálcica caracterizada por calcificação, que se iniciava na base das cúspides e se dirigia para a borda das mesmas; e 3) na valva aórtica bicúspide, visibilização de apenas dois folhetos com espessamento das cúspides.

A doença arterial coronariana foi avaliada por critérios angiográficos de lesões obstrutivas $\geq 50 \%$ para o tronco (TC) da coronária esquerda e $\geq 70 \%$ nas seguintes artérias: descendente anterior (DA), coronária direita (CD) e circunflexa (CX). Para as artérias marginais (MG) e diagonais (DG) ou mesmo um ramo intermediário de importância anatômica (diagonalis - DI) foram considerados como critérios lesões $\geq 70 \%$ e artérias com diâmetro maior que $3 \mathrm{~mm}$. Embora apenas as lesões graves, de natureza anatomicamente cirúrgica, tenham sido consideradas neste trabalho, as lesões leves e moderadas foram classificadas para fins de registro, também por critérios angiográficos. Foram caracterizadas como lesões leves aquelas com obstruções $<50 \%$ da luz do vaso e como lesões moderadas as que apresentavam obstruções da luz do vaso entre $50 \%$ e $70 \%{ }^{7}$.

As variáveis estudadas foram: idade, sexo, índice de massa corporal (IMC, sendo considerados normais os pacientes com IMC $<25 \mathrm{~kg} / \mathrm{m}^{2}$; com sobrepeso, pacientes com IMC entre $25 \mathrm{~kg} / \mathrm{m}^{2}$ e $30 \mathrm{~kg} / \mathrm{m}^{2}$; e obesos, pacientes com IMC > $30 \mathrm{~kg} / \mathrm{m}^{2}$ ), dor torácica (típica, atípica e ausência de dor), dispnéia, tabagismo e carga tabágica (número de anos que o paciente fumou um maço por dia), hipertensão arterial sistêmica (HAS, considerada pressão arterial sistólica $\geq 140$ $\mathrm{mmHg}$ ou pressão arterial diastólica $\geq 90 \mathrm{mmHg}$ ou uso de medicação anti-hipertensiva), doença arterial coronariana, história familiar e diagnósticos relacionados a essa doença, diabete melito (considerado quando a glicemia de jejum apresentasse valores $\geq 126 \mathrm{mg} / \mathrm{dl}$ ) e dislipidemia (considerada quando o nível de colesterol total era $\geq 200 \mathrm{mg} / \mathrm{dl} \mathrm{e} /$ ou o de triglicerídeos era $\geq 150 \mathrm{mg} / \mathrm{dl}$ ).

Na análise estatística, foram utilizados: o teste do quiquadrado para a comparação das proporções e o teste $t$ de Student para a análise das variáveis contínuas, sendo p considerado significativo quando $\leq 0,05$; o modelo de regressão logística para estimar a influência das variáveis selecionadas sobre o desfecho, ou seja, presença de doença arterial coronariana; e o modelo Log linear para analisar relações de interdependência de primeira, segunda e terceira ordens entre as variáveis. O programa estatístico utilizado foi o sistema R, versão 2.3. Um banco de dados (Access) foi criado com as informações clínicas e dos exames complementares dos pacientes.

A pesquisa foi aprovada pelo Comitê de Ética em Pesquisa do Instituto Estadual de Cardiologia Aloysio de Castro (Rio de Janeiro, RJ), em 5/10/2005. 


\section{Artigo Original}

\section{Resultados}

1) Análise dos grupos com cardiopatia valvar reumática e não-reumática

A tabela 1 compara as variáveis dos grupos com cardiopatia valvar reumática e não-reumática. As variáveis que apresentaram diferenças estatísticas foram: sexo (predominantemente feminino no grupo de reumáticos e masculino no grupo de não-reumáticos); idade (maior entre os não-reumáticos); dor torácica tipicamente anginosa (mais freqüente no grupo de não-reumáticos); ausência de dor torácica (maior freqüência no grupo de reumático); HAS, diabete melito, dislipidemias e presença de doença arterial coronariana (mais freqüentes no grupo de não-reumáticos). Por outro lado, não houve diferença significativa entre os dois grupos quanto às variáveis: IMC (normal, sobrepeso e obesidade); dor torácica atípica; dispnéia; história familiar de doença arterial coronariana; tabagismo e ex-tabagismo. Esses dois grupos, portanto, apresentavam características clínicas heterogêneas.

2) Relação da doença arterial coronariana com as demais variáveis

Para examinar a influência da etiologia da valvopatia na determinação da presença de doença arterial coronariana, foi realizada análise utilizando-se o modelo de regressão logística, em que a doença era considerada a variável dependente e as demais tomadas eram consideradas determinantes. As variáveis incluídas nesta análise foram: sexo, idade, IMC (sobrepeso e obesidade), dor torácica típica, HAS, diabete melito, dislipidemia, tabagismo, e etiologia reumática.
Considerando a mediana de 54 anos, o corte para a idade foi: $<55$ e $\geq 55$ anos. A etiologia reumática não demonstrou ser uma variável determinante na doença arterial coronariana, permitindo proceder à análises envolvendo os 294 pacientes valvopatas, independentemente da etiologia (tab. 2). Dada sua importância clínica na doença arterial coronariana, as variáveis tabagismo e sexo foram mantidas no modelo, apesar de não apresentarem significância estatística. As que se relacionaram significativamente com a doença no modelo final da análise foram: idade, dor torácica típica, HAS, diabete melito e dislipidemia, como mostrado na expressão final do modelo de regressão logística:

Logito $(\mathrm{DAC})=-5,07+0,78$ sexo $+0,04$ idade $+2,05$ dor torácica típica $+0,91$ hipertensão arterial sistêmica $+2,04$ diabete $+1,64$ dislipidemia $+0,57$ tabagismo, onde:

Logito $(\mathrm{DAC})=\operatorname{Ln}\{\operatorname{Pr}(\mathrm{DAC}=1) / \operatorname{Pr}(\mathrm{DAC}=0)\}$.

3) Relações de interdependência entre doença arterial coronariana e as demais variáveis

Para sustentar os resultados obtidos na regressão logística, foi utilizado também o modelo Log linear, que examinou relações de interdependência entre as variáveis. Trata-se de um modelo de análise multivariada, que se caracteriza por tratar todas as variáveis com o mesmo nível de importância, e não como de desfecho e explicativas. As variáveis que isoladamente se mostraram significativas no modelo foram: doença arterial coronariana, sexo, idade, dor torácica típica, HAS, diabete melito e dislipidemia. Na relação de interdependência (interação) de segunda ordem, foi encontrada associação de doença arterial coronariana com

Tabela 1 - Características do grupo de reumáticos e do grupo de não-reumáticos

\begin{tabular}{|c|c|c|c|c|c|c|}
\hline & Variáveis & $\begin{array}{l}\text { Reumáticos } \\
(n=175)\end{array}$ & $\%$ & $\begin{array}{l}\text { Não-reumáticos } \\
(n=119)\end{array}$ & $\%$ & Valor de $p$ \\
\hline \multirow{2}{*}{ Sexo } & Masculino & 54 & 30,86 & 85 & 71,43 & $<0,0001$ \\
\hline & Feminino & 121 & 69,14 & 34 & 28,57 & $<0,0001$ \\
\hline \multirow[t]{2}{*}{ Idade } & (média) & 50,87 & & 63,66 & & \\
\hline & Normal & 147 & 84,00 & 89 & 74,79 & 0,072 \\
\hline \multirow[t]{3}{*}{ IMC } & Sobrepeso & 22 & 12,57 & 23 & 19,33 & 0,157 \\
\hline & Obesidade & 6 & 3,43 & 7 & 5,88 & 0,470 \\
\hline & Dor típica & 12 & 6,86 & 57 & 47,90 & $<0,0001$ \\
\hline \multirow[t]{8}{*}{ Dor torácica } & Dor atípica & 34 & 19,43 & 22 & 18,49 & 0,959 \\
\hline & Ausência de dor & 129 & 73,71 & 40 & 33,61 & $<0,0001$ \\
\hline & HAS & 52 & 29,71 & 70 & 58,82 & $<0,0001$ \\
\hline & Diabete & 7 & 4,00 & 17 & 14,29 & 0,003 \\
\hline & Dislipidemia & 14 & 8,00 & 21 & 17,65 & 0,020 \\
\hline & $\mathrm{DAC}$ & 7 & 4,00 & 40 & 33,61 & $<0,0001$ \\
\hline & História familiar & 76 & 43,43 & 66 & 55,46 & 0,056 \\
\hline & $\begin{array}{l}\text { Tabagismo e } \\
\text { ex-tabagismo }\end{array}$ & 87 & 49,71 & 58 & 48,73 & 0,789 \\
\hline
\end{tabular}

n- número de pacientes; IMC - índice de massa corporal; HAS - hipertensão arterial sistêmica; DAC - doença arterial coronariana. 


\section{Artigo Original}

Tabela 2 - Características demográficas e clínicas da população total

\begin{tabular}{|c|c|c|c|}
\hline Variáveis & & $\mathrm{n}$ & $\%$ \\
\hline \multirow{4}{*}{ Idade } & $\geq 55$ & 146 & 49,65 \\
\hline & $<55$ & 148 & 50,34 \\
\hline & Média & 56 & \\
\hline & Mediana & 54 & \\
\hline \multirow{2}{*}{ Sexo } & Masculino & 139 & 47,30 \\
\hline & Feminino & 155 & 52,70 \\
\hline IMC & $\begin{array}{l}\text { Sobrepeso e } \\
\text { obesidade }\end{array}$ & 58 & 19,72 \\
\hline \multirow{3}{*}{ Dor torácica } & Típica & 69 & 23,46 \\
\hline & Atípica & 56 & 19,04 \\
\hline & Ausência de dor & 169 & 57,48 \\
\hline \multirow{6}{*}{$\begin{array}{l}\text { Variáveis clínicas, da } \\
\text { história e laboratoriais }\end{array}$} & HAS & 122 & 41,49 \\
\hline & Diabete & 24 & 8,16 \\
\hline & Dislipidemia & 35 & 11,90 \\
\hline & DAC & 47 & 15,98 \\
\hline & História familiar & 142 & 48,29 \\
\hline & $\begin{array}{l}\text { Tabagismo e } \\
\text { ex-tabagismo }\end{array}$ & 145 & 49,31 \\
\hline
\end{tabular}

n - número de pacientes; IMC - índice de massa corporal; HAS - hipertensão arterial sistêmica; DAC - doença arterial coronariana.

sexo masculino, idade $\geq 55$ anos, dor torácica típica, HAS, diabete e dislipidemia; associação entre idade $\geq 55$ anos e dor torácica típica; idade $\geq 55$ anos e HAS; dor torácica típica e diabete; HAS e diabete; diabete e dislipidemia. Na relação de interdependência de terceira ordem, foi observada associação de doença arterial coronariana com dor torácica típica e diabete, com HAS e diabete e, ainda, com diabete e dislipidemia. Assim, as variáveis que mais fortemente se associaram à doença arterial coronariana foram: dor torácica típica, diabete melito e dislipidemia (fig. 1).

4) Características clínicas da população com e sem doença arterial coronariana

Na tabela 3 encontram-se as características clínicas da população segundo a presença e a ausência da doença arterial coronariana. Os achados são resumidos como se segue.

Na faixa etária $\geq 55$ anos, o porcentual de prevalência da doença foi maior que na faixa etária $<55$ anos. A freqüência da doença foi maior no sexo masculino que no sexo feminino. No grupo com presença da doença, foram observados porcentuais significativamente maiores nas seguintes variáveis: dor torácica típica, HAS, diabete, dislipidemia, estenose aórtica, e índice de carga tabágica. No grupo com ausência da doença foram observados porcentuais significativamente maiores nas variáveis ausência de dor e estenose mitral. Não houve diferença significativa entre os

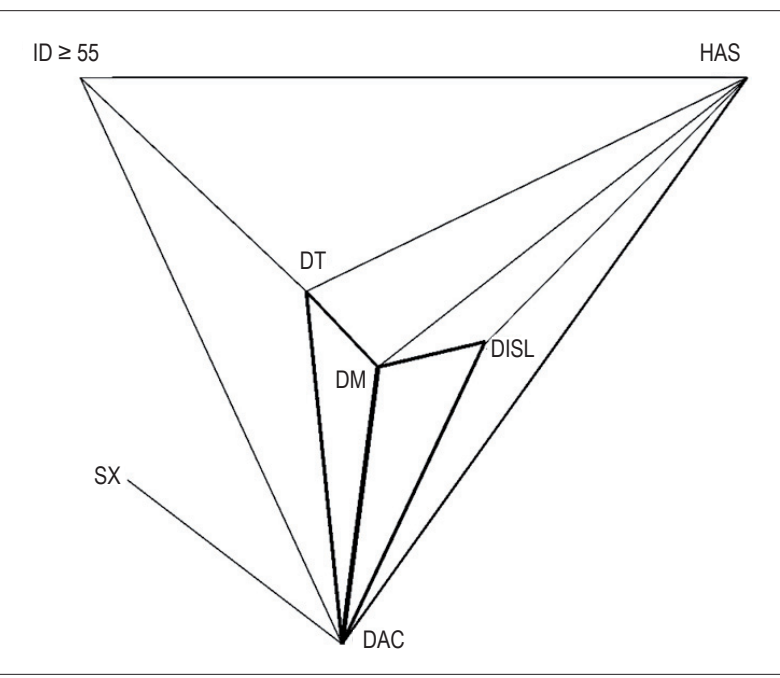

Fig. 1 - Interdependência dos fatores de risco para doença arterial coronariana na faixa etária > 55 anos. DAC - doença arterial coronariana; DISL - Dislipidemia; DM - Diabete melito; DT - Dor torácica típica; ID - Idade; SX - Sexo; HAS - hipertensão arterial sistêmica.

grupos quanto às seguintes variáveis: sobrepeso e obesidade, história familiar, dor atípica, tabagismo e ex-tabagismo (embora porcentualmente maior no grupo com doença arterial coronariana que no grupo sem a doença).

\section{Discussão}

Neste estudo, foi examinada a prevalência da doença arterial coronariana de etiologia reumática e não-reumática em pacientes com idade $\geq 40$ anos. Em sua forma grave, a doença foi considerada não apenas quando havia lesão de tronco e lesões trivasculares, que afetavam simultaneamente as principais artérias coronárias, mas também lesões uni e bivasculares e lesões graves, que afetavam ramos secundários de importância anatômica. Os critérios deste estudo parecem bastante abrangentes, levando-se em conta que a maioria dos estudos revisados considera lesão grave do tronco quando $\geq$ $50 \%$, e lesões das artérias coronárias (CD, CX e DA) quando > $70 \%{ }^{8,9}$. Para alguns autores ${ }^{10}$, a condição ideal para indicação cirúrgica de revascularização miocárdica é aquela em que os pacientes exibem lesões proximais de pelo menos $70 \%$ nas artérias coronárias principais com boas porções distais.

Segundo os achados deste estudo, é possível identificar clinicamente pacientes orovalvares com menor probabilidade de apresentar doença arterial coronariana; com base nisso, os autores sugerem que o estudo coronariográfico não seja indicado indiscriminadamente para todos os pacientes, mas para aqueles que apresentem evidências clínicas claras e fatores preditivos da doença.

No que se refere à etiologia, observou-se baixa prevalência de doença arterial coronariana grave entre os pacientes reumáticos ${ }^{11}$, ponderando-se inicialmente que essa etiologia poderia conferir algum grau de proteção para o desenvolvimento da aterosclerose coronariana, como notado por outros investigadores $^{12}$. A impressão se baseava no fato de que, 


\section{Artigo Original}

Tabela 3 - Características clínicas da população com DAC e sem DAC

\begin{tabular}{|c|c|c|c|c|c|}
\hline \multirow{2}{*}{ Variáveis } & \multicolumn{2}{|c|}{ Sem DAC } & \multicolumn{2}{|c|}{ Com DAC } & \multirow{2}{*}{ Valor de $p$} \\
\hline & $(n=247)$ & $\%$ & $(n=47)$ & $\%$ & \\
\hline Idade $\geq 55$ anos & 107 & 43,32 & 39 & 82,96 & $<0,0001$ \\
\hline Idade $<55$ anos & 140 & 56,68 & 8 & 17,02 & $<0,0001$ \\
\hline Sexo masculino & 106 & 42,91 & 33 & 70,21 & 0,0027 \\
\hline Sexo feminino & 141 & 57,09 & 14 & 29,79 & 0,0027 \\
\hline Sobrepeso e obesidade & 47 & 19,03 & 11 & 23,40 & 0,79 \\
\hline Dor torácica típica & 37 & 14,98 & 32 & 68,09 & $<0,0001$ \\
\hline Dor torácica atípica & 49 & 19,84 & 7 & 14,89 & 0,73 \\
\hline Ausência de dor & 161 & 65,18 & 8 & 17,02 & $<0,0001$ \\
\hline HAS & 90 & 36,44 & 32 & 68,09 & 0,0003 \\
\hline Diabete & 11 & 4,45 & 13 & 27,66 & $<0,0001$ \\
\hline Dislipidemia & 22 & 8,91 & 13 & 27,66 & 0,0013 \\
\hline História familiar & 115 & 46,56 & 27 & 57,45 & 0,30 \\
\hline Tabagismo e ex-tabagismo & 116 & 46,96 & 29 & 61,70 & 0,18 \\
\hline Estenose aórtica & 58 & 23,48 & 31 & 65,96 & $<0,0001$ \\
\hline Estenose mitral & 78 & 31,58 & 4 & 8,51 & $<0,0001$ \\
\hline Carga tabágica ( $n^{\circ}$ de anos que fumou um maço por dia) & 12 & & 27,2 & & $<0,0001$ \\
\hline
\end{tabular}

DAC - doença arterial coronariana; $n$ - número de pacientes; HAS - hipertensão arterial sistêmica.

como de modo geral esses pacientes se submetem a profilaxia de febre reumática com o uso de antibiótico intramuscular a cada 21 dias durante muitos anos, isso poderia ter efeito protetor antibacteriano e antiinflamatório na gênese da aterosclerose coronariana. Entretanto, os achados deste estudo não corroboraram a idéia. Uma revisão das histórias clínicas demonstrou que a maioria dos pacientes nunca fez profilaxia para febre reumática, o que parece fazer sentido, pois se de fato isso tivesse ocorrido de modo adequado certamente suas valvas cardíacas não chegariam a um estado tal de destruição como comumente encontrado. Os dados apontaram para uma explicação alternativa: a de que a baixa prevalência poderia decorrer das características demográficas e clínicas dessa população ${ }^{13,14}$. Em um grupo predominantemente feminino e jovem e, portanto, com menos co-morbidades, é razoável que a prevalência da doença seja menor que em outro predominantemente masculino com idade mais avançada.

A prevalência da doença arterial coronariana foi muito superior no grupo de não-reumáticos, que se caracterizava por predominância do sexo masculino e idade mais avançada ${ }^{15,16}$, ao contrário do observado no grupo de reumáticos. Outras características clínicas diferiram significativamente entre esses dois grupos (dor torácica tipicamente anginosa e fatores de risco como hipertensão arterial sistêmica, diabete e dislipidemia) ${ }^{17,18}$, mais encontradas no grupo de nãoreumáticos, sendo a estenose aórtica por degeneração fibrocálcica o diagnóstico mais freqüente nesse grupo ${ }^{19,20}$ e a estenose mitral mais freqüente no grupo de reumáticos ${ }^{13}$.

Os aspectos demográficos e clínicos que caracterizaram os pacientes do grupo com doença arterial coronariana foram (tab. 3): idade $\geq 55$ anos, sexo masculino, dor torácica típica, HAS, diabete, dislipidemia, carga tabágica e presença de dois ou mais fatores de risco, sendo estenose aórtica o diagnóstico mais associado à doença. $\mathrm{O}$ diagnóstico menos associado à doença arterial coronariana foi a estenose mitral. Dados semelhantes foram descritos em outros estudos , $^{91,22}$.

Alguns aspectos observados nos registros do banco de dados utilizado neste estudo merecem ser notados. Poucos portadores de doença arterial coronariana estavam na faixa etária entre 40 e 60 anos, mas apresentavam clínica que sugeria a presença da doença. Assim, é pouco provável que não fossem notados em uma estratificação clínica. Abaixo dos 60 anos, a prevalência da doença foi de apenas 1,7\%, ou seja, onde existe maior concentração de pacientes com valvopatia reumática ela é quase inexistente, o que pode questionar a validade de se submeter essa população a coronariografia de modo indiscriminado. Independentemente da etiologia, a prevalência da doença foi de $6,95 \%$ entre os pacientes com idade $<60$ anos, aumentando para 31,8\% entre aqueles com idade $\geq 60$ anos, o que é natural, uma vez que, com o avanço da idade, aumentam as co-morbidades. Em uma análise comparativa entre ausência de fatores de risco e presença de um, dois, três, quatro e cinco fatores de risco, respectivamente, observa-se que os pacientes apresentando dois ou mais fatores de risco concentraram-se predominantemente no grupo com doença arterial coronariana, enquanto no grupo sem a doença foram observados maiores porcentuais de pacientes com apenas um ou sem fatores de risco. Assim, o estudo hemodinâmico poderia ser evitado nos pacientes sem angor típico e sem fatores de risco cardiovascular associados. Com respeito às lesões coronarianas, também valem algumas 
considerações. De acordo com os registros deste estudo, entre 187 pacientes abaixo de 60 anos apenas 1 (0,53\%) apresentava lesão do tronco e 2 (1,06\%) apresentavam lesão trivascular grave. Abaixo dessa idade, foram encontrados apenas 3 pacientes anatômica e verdadeiramente cirúrgicos, apresentando angor típico e associação de dois ou mais fatores de risco, e, portanto, seguramente não passariam despercebidos por uma boa estratificação clínica.

Na literatura, a indicação de cateterismo pré-operatório de rotina tem nível de evidência $C^{4}$, o que significa que essa é uma evidência baseada apenas na opinião de especialistas, em estudo de casos ou em medida tomada como precaução, não havendo outro fundamento mais bem estabelecido. Por exemplo, pacientes com insuficiência aórtica grave não apresentando sintomas de isquemia miocárdica ou fatores de risco que sabidamente aumentam a prevalência de doença arterial coronariana poderiam ter a coronariografia préoperatória evitada com segurança, de acordo com alguns pesquisadores ${ }^{23}$. Outros ${ }^{24}$ correlacionam baixa prevalência da doença e valvopatia, chamando a atenção para o fato de que a coronariografia é desnecessária nesses pacientes, exceto na presença de fatores de risco ou achados clínicos como angina e infarto do miocárdio prévio.

\section{Conclusões}

A nosso ver, a indicação rotineira de coronariografia pré-operatória baseada apenas no critério de idade precisa ser repensada. A coronariografia diagnóstica, embora sendo método com baixa mortalidade, apresenta índice de complicações que varia desde menos de $1 \%$ até próximo de $5 \%$. Entretanto, quando tais complicações existem, podem ter efeito adverso bastante significativo. Entre outras complicações, destaca-se a ocorrência de lesões cerebrais por microembolias gasosas e sólidas, que pode acarretar comprometimento cognitivo dos pacientes, particularmente os predispostos ${ }^{25}$.

A cirurgia de revascularização miocárdica, quando analisada a partir de banco de dados que expressam a realidade da prática clínica, apresenta índices de mortalidade acima de 3,5\% nos Estados Unidos ${ }^{26,27}$. No Brasil, esses índices estão acima de 5\%. No Rio de Janeiro, a letalidade hospitalar na cirurgia de revascularização miocárdica varia de 4,8\%, entre homens com diagnóstico de doença isquêmica crônica, a $14 \%$ entre mulheres com angina ${ }^{28}$. Nas cirurgias conjuntas, os índices chegam a ser cinco vezes maiores que na cirurgia valvar isolada ${ }^{29}$. Em estudo comparando pacientes previamente revascularizados com aqueles que não se submeteram ao procedimento, não foi encontrada diferença significativa na evolução dos dois grupos ${ }^{30}$. Os autores não consideram vantagem submeter pacientes candidatos a cirurgia vascular de grande porte, portadores de doença arterial coronariana, a cirurgia cardíaca e mesmo a angioplastia pré-operatória. A nosso ver, a mesma consideração se aplica à investigação de doença arterial coronariana em pacientes valvopatas com baixa probabilidade de apresentar a doença, especialmente porque o estudo hemodinâmico carrega risco adicional, muitas vezes com maior probabilidade de complicações que a própria doença. Entretanto, considerase razoável a indicação de revascularização miocárdica no momento da troca valvar quando essa indicação é feita para os pacientes que apresentam evidências clínicas de isquemia, que se traduzem pela presença de angina de peito associada aos fatores preditivos de doença arterial coronariana. Outro aspecto importante é que a indicação de cirurgia de revascularização associada a troca valvar com freqüência é de caráter "profilático", uma vez que, em geral, a doença arterial coronariana é aleatoriamente explorada e casualmente encontrada. Em muitos casos, a indicação cirúrgica se faz pelo fato de uma determinada lesão ter sido descoberta na coronariografia. Uma vez que a indicação por causa da lesão seja feita, aproveita-se para revascularizar o paciente.

A abordagem deste estudo baseia-se no fato de não existirem evidências robustas de que tal conduta beneficia o paciente. Ao contrário, o que se observa é que o manuseio da troca valvar associado ao procedimento de revascularização miocárdica aumenta de modo significativo a mortalidade a curto e médio prazos.

\section{Potencial Conflito de Interesses}

Declaro não haver conflito de interesses pertinentes.

\section{Fontes de Financiamento}

O presente estudo não teve fontes de financiamento externas.

\section{Vinculação Acadêmica}

Este artigo é parte de tese de doutorado de Dany David Kruczan pela Universidade Federal do Rio de Janeiro.

\section{Referências}

1. Pomerantzeff PM, Barbosa GV, Sousa Fo BS, Brandão CMA, Ribeiro EJ, Costa FDA, et al. Diretrizes para a conduta dos pacientes com doenças das valvas cardíacas. In: Portal Cardiol [online]; 2003. [citado 2006 ago 20]. Disponível em: http://publicacoes.cardiol.br/consenso/2003/site/036.asp.

2. American College of Cardiology. American Heart Association. Task Force on Practice Guidelines (Writing Committee to revise the 1988 guidelines for the management of patients with valvular heart disease). Society of

\footnotetext{
Cardiovascular Anesthesiologists. Bonow RO, Carabello BA, Chattergee K, de Leon AC Jr, Faxon DP, Freed MD, et al. ACC/AHA 2006 guidelines for the management of patients with valvular heart disease: a report of the American College of Cariology/AHA Task Force on Practice guidelines. J Am Coll Cardiol. 2006; 48 (3): e1-148.

3. Argüelles E, Fiszman P, Fakoury L. Febre reumática e doenças valvulares do coração. Rio de Janeiro: Intermédica, 1984.
} 


\section{Artigo Original}

4. Terreri MT, Len C, Hilário MOE, Goldenberg MB. Utilização de recursos e custos de pacientes com febre reumática. Rev Bras Reumatol. 2002; 42 (4): 211-7.

5. Azevedo AC, Sekeff J. Cardiologia clínica. São Paulo: Sarvier, 1994.

6. Suaide Silva CE, Ferreira LDC, Monaco CG, Gil MA, Peixoto LB, Leal SMB, et al. O ecocardiograma no apoio à decisão clínica. 3a. ed. São Paulo: Revinter, 2003

7. King III. SB, Douglas JS Jr. Atlas of heart diseases. New York: McGraw Hill; 1985.

8. Marchand E, Pichard, A, Casanegra P. Association of coronary artery disease and valvular heart disease in Chile. Clin Cardiol. 1983; (6): 352- 6.

9. Savova A, Stoitchev S, Ovanesyan H, Finkov B, Belov J. Coronary artery disease in patients with valvular heart disease. Z. Cardiol. 1986; 75 (Suppl 2): $73-5$.

10. Sousa JEMR, Batlouni M, Jatene AD. Insuficiência coronária. São Paulo: Sarvier, 1984

11. Rangel A, Hernández J, Iris JM, Badui E. Indicacion de la coronariografia en las valvulopatias cardiacas. Arch Inst Cardiol Méx. 1996; 66: 60-9.

12. Jose VJ, Gupta SN, Joseph G, Chandy ST, George NJ. Prevalence of coronary artery disease in patients with rheumatic heart disease in the current era. Indian Heart J. 2004; 56 (2): 129-31.

13. Chu PH, Chiang CW, Hsu LA, Lin KH, Cheng NJ. Low prevalence of coronary arterial disease in Chinese adult with mitral stenosis. Chang Gung Med J. 2001; 24 (2): 97-102.

14. Guray Y, Guray U, Yilmaz BM, Mecit B, Kisacik H, Korkmaz S. Prevalence of angiographically significant coronary artery disease in patients with rheumatic mitral stenosis. Acta Cardiol. 2004; 59 (3): 305-9.

15. Alexopoulos D, Kolovou G, Kyriakidis M, Antonopoulos A, Adamopavlos S, Sleight P. Angina and coronary artery disease in patients with aortic valvular disease. Angiology. 1993; 44 (9): 707-11.

16. Bozbas H, Yildirir A, Küçük MA, Ozqül A, Atar I, Sezgin A, et al. Prevalence of coronary artery disease in patients undergoing valvular surgery due to rheumatic involvement. The Anadolu Kardiyol Derq. 2004; 4: 223-6.

17. Ramsdale DR, Faragher EB, Bennett DH, Bray CL, Ward C, Beton DC. Preoperative prediction of significant coronary artery disease in patients with valvular heart disease. Br Med J. 1982; 284: 223-6.

18. Gomez Doblas JJ, Jimenez Navarro M, Rodriguez Bailón I, Alonso Briales JH,
Hernandez Garcia JM, Montiel Trujillo A. Coronariografia preoperatoria en pacientes valvulares: análisis de probabilidad de lesión coronária. Rev Esp Cardiol. 1998; 51 (9): 756-61.

19. Mandal AB, Gray IR. Significance of angina pectoris in aortic valve stenosis. Br Heart J. 1976; 38: 811-5.

20. Rapp AH, Hillis LD, Lange RA, Cigarroa JE. Prevalence of coronary artery disease in patients with aortic stenosis with and without angina pectoris. Am J Cardiol. 2001; 87 (10): 1216-7.

21. Sheiban I, Trevi GP, Benussi P, Marini A, Accardi R, Di Bona E, et al. Incidence of coronary artery disease in patients with valvular heart disease. Z.Cardiol. 1986; 75 (Suppl 2): 76-9.

22. Raungratanaamporn O, Srivanasont N, Chaithiraphan S, Bhuripanyo K. Prevalence of coronary artery disease in patients with valvular heart disease. J Med Assoc Thai. 1995; 78 (1): 1-4.

23. Timmermans $\mathrm{P}$, Willems JL, Piessens J, De Geest $\mathrm{H}$. Angina pectoris and coronary artery disease in severe aortic regurgitation. Am J Cardiol. 1988; 61 (10): 826-9.

24. Meruane SJ, Kauffmann QR, Florenzano FF. Associacion de enfermedad coronaria y valvulopatias: implicaciones para la indicacion de coronariografia. Rev Med Chil. 1989; 117 (6): 641-6.

25. Lund C, Nes RB, Ugelstad TP, Due-Tennesen P, Andersen R, Hol PK, et al. Cerebral emboli during left heart catheterization may cause acute brain injury. Eur Heart J. 2005; 26 (13): 1269-75.

26. Clark RE. Outcome as a function of annual coronary artery bypass graft volume. Ann Thorac Surg. 1996; 61: 21-6.

27. Tu JV, Sykora K, Naylor CD. Assessing the outcomes of coronary artery bypass graft surgery: how many risk factors are enough. J Am Coll Cardiol. 1997; 30: 1317-23.

28. Godoy PH, Klein $\mathrm{CH}$, Souza e Silva NA, Oliveira GMM, Fonseca TMP Letalidade na cirurgia de revascularização do miocárdio no Estado do Rio de Janeiro - SIH/SUS - no Período 1999-2003. Rev SOCERJ. 2005; 18: 123-30.

29. Herlitz J, Brandrup-Wognsen G, Caidahl K, Haglid M, Karlsson T, Albertsson P, et al. Mortality and morbidity among patients who undergo combined valve and coronary artery bypass surgery: early and late results. Eur J Cardiothorac Surg. $1997 ; 12: 836-46$.

30. McFalls EO, Ward HB, Moritz TE, Goldman S, Krupski WC, Littooy F, et al. Coronary-artery revascularization before elective major vascular surgery. $\mathrm{N}$ Engl J Med. 2004; 351 (27): 2795-804. 